\title{
Anti-HLA-A2/NY-ESO-1 TCR-transduced Autologous T Lymphocytes
}

National Cancer Institute

\section{Source}

National Cancer Institute. Anti-HLA-A2/NY-ESO-1 TCR-transduced Autologous T

Lymphocytes. NCI Thesaurus. Code C122679.

Autologous human peripheral blood T-lymphocytes transduced with a lentiviral or retroviral vector encoding a human leukocyte antigen A2 (HLA-A2) restricted anti-cancertestis antigen 1 (NY-ESO-1) T-cell receptor (TCR) gene, with potential antineoplastic activity. Following leukapheresis, isolation of lymphocytes, expansion ex vivo, transduction, and re-introduction into the patient, the anti-HLA-A2/NY-ESO-1 TCRtransduced autologous T lymphocytes recognize and bind to NY-ESO-1/HLA-A2-positive tumor cells. This results in cytotoxic T-lymphocyte (CT L)-mediated elimination of NYESO-1-positive cancer cells. NY-ESO-1, a tumor-associated antigen (TAA), is found in normal testis and on the surface of various tumor cell types. 